\title{
DANOS CAUSADOS PELA INFECÇÃO DE OÍDIO EM DIFERENTES ESTÁDIOS FENOLÓGICOS DA SOJA
}

\author{
S. Igarashi ${ }^{1}$, G.M. Oliveira ${ }^{1}$, L.C.M. Camargo ${ }^{1}$, J. Falkoski Filho ${ }^{1}$, C.G. Gardiano ${ }^{1}$, M.G. Balan ${ }^{2}$
}

${ }^{1}$ Universidade Estadual de Londrina, Centro de Ciências Agrárias, Departamento de Agronomia, Laboratório de Fitopatologia, CP 6001, CEP 86051-990, Londrina, PR, Brasil. E-mail: sigarashi@uel.br

\section{RESUMO}

Ainda não há estudos precisos que quantifiquem os prejuízos decorrentes de infecção por oídio e/ou outras doenças foliares, para a maioria das culturas de importância econômica no Brasil. O objetivo foi quantificar as perdas causadas por oídio (Microsphaera diffusa) infectando a cultura da soja em diferentes estádios fenológicos e relacioná-las ao desenvolvimento e produtividade da cultura. O experimento foi desenvolvido em ambiente protegido, e os tratamentos foram testemunha controlada, testemunha sem controle, infecção iniciada em $R_{1}-R_{2}$, infecção iniciada em $R_{3}-R_{4^{\prime}}$ infecção iniciada em $R_{5.1}-R_{5.2}$ e infecção iniciada em $R_{5.3}-R_{5.4}$. A avaliação foi feita semanalmente, considerando a porcentagem da área foliar infectada. Os resultados mostraram que, no tratamento em que houve infecções iniciadas em $R_{1}-R_{2}$ e $R_{3}-R_{4}$, a porcentagem de área foliar afetada foi maior (41\% e 38\%, respectivamente), com consequente menor produtividade $(1.186,6$ e $1.309,5 \mathrm{~kg} \cdot$ ha $^{-1}$ respectivamente). No tratamento em que a infecção ocorreu em $R_{5.3}-R_{5.4^{\prime}}$, houve a menor média de área foliar afetada pela doença (24\%) e a produtividade teve queda de $26 \%$. Os resultados mostraram que as perdas de produtividade pelo oídio na cultivar Embrapa 48 variaram ao redor de 26 a 50\%, e que a recomendação oficial para o início de controle do oídio da soja, quando esta se apresentar entre 40 e 50\% de severidade, deve ser questionada e outros trabalhos neste âmbito devem ser desenvolvidos para determinação das perdas ocasionadas por esta doença na cultura.

PALAVRAS-CHAVE: Microsphaera diffusa, rendimento, Glycine Max.

\section{ABSTRACT}

DAMAGES CAUSED BY INFECTION OF POWDERY MILDEWS IN DIFFERENT PHENOLOGICALSTAGES OF SOYBEANS. At present there are no precise studies quantifying the damages caused by powdery mildews and other foliar diseases for the majority of economically important crops in Brazil. The objective of the present study was to quantify the losses caused by powdery mildews (Microsphaera diffusa) in soybeans in different phenological stages, and to correlate them with the development and yield of the crop. The trials was carried out in the greenhouse and the treatments were: controlled check, noncontrolled check, infection initiated at stage R1-R2, infection initiated at stage R3-R4, infection initiated at stage R5.1-R5.2, infection initiated at stage R5.3-R5.4. The evaluation was done weekly considering the percentage of infected leaf area. The results showed that for the infection beginning at stages R1-R2 and R3-R4 the percentage of affected leaf area was higher (41\% and 38\%), with consequently lower yields $(1,200$ and 1,240 $\mathrm{kg} \mathrm{ha}^{-1}$ ). When the infection occurred later at stage R5.3-R5.4, a lower affected leaf area (24\%) was observed, and the yield decreased $26 \%$. The results showed that the loss of yield by powdery mildew in cultivar Embrapa 48 ranged from around 26 to 50\%, and that the official recommendation for the beginning of control of powdery mildew of soybean, where it presents between 40 and $50 \%$ of severity, should be questioned, and other work in this area should be undertaken to determine the loss caused by this disease in the crop.

KEY WORDS: Microsphaera diffusa, yield, Glycine max.

\section{INTRODUÇÃO}

Dentre os países produtores de soja, o Brasil se destaca como o segundo maior produtor mundial, perdendo apenas para os EUA, e como o maior exportador mundial, desde 2003. Atualmente, a região Centro-Oeste é a maior produtora de grãos $(45,9 \%)$, seguida pela regiãoSul $(38,3 \%)$ epelas demais $(15,8 \%)$

${ }^{2}$ Faculdade Integrado de Campo Mourão, Campo Mourão, PR, Brasil. 
(COMPANHIA Nacional de Abastecimento, 2007). Vários fatores limitam a obtenção de altos rendimentos na cultura da soja, entre eles, as doenças foliares são consideradas um dos principais, pois afetam a qualidade de grãos e causam perdas consideráveis no rendimento final, sendo também de difícil controle.

Atualmente, com a abertura de novas fronteiras agrícolas e com a expansão do cultivo da soja nessas áreas, sem a adoção de critérios técnicos adequados, houve rápida disseminação do complexo de doenças na cultura da soja no Brasil. A ocorrência de modo epidêmico dessas doenças tem influenciado significativamentea qualidadedegrãosea produtividadee, por conseguinte, o aumento no custo de produção de soja, principalmente pela necessidade maior do uso de fungicidas. Entre essas doenças podemos destacar a ferrugem asiática (Phakopsora pachyrhizi), a antracnose (Colletotrichum truncatum var. trucata), o oídio (Microsphaeradiffusa), omíldio(Peronosporamanshurica), as doenças de final de ciclo(Septoriaglycines eCercospora kikuchii), a murcha de esclerócio (Sclerotium rolfsii), a mancha alvo(Corynespora cassiicola) e a mela ou requeima da soja (Rhizoctonia solani). Todavia, a importância econômica de cada uma dessas doenças varia de ano para ano e de região para região, principalmente em função da cultivar, das condições do ambientee do tipo de tecnologia adotado (EMBRAPA SoJA, 2002).

O oídio (Microsphaera diffusa Cooke \& Peck) da soja, segundo SARTORATO; YORINORI (2001), é uma das doenças mais antigas dessa leguminosa, sendo que o primeiro registro ocorreu na Alemanha, em 1921. Desdeentão, há referências de ocorrência em praticamente todas as regiões produtoras no mundo, inclusive no Brasil (SINCLAIR, 1999). Foi identificado primeiramente em casa-de-vegetação e a campo, em final de ciclo de cultivar tardia, sem causar danos significativos. A constatação de oídio a campo só ocorreu no ano de 1945 (LehMAN, 1947). Atualmente, existem mais de 20 espécies de oídio que causam prejuízo econômico reportados na agricultura brasileira.

O fungo pode instalar-se em qualquer estádio de desenvolvimento da cultura, desenvolvendo seu micélio na superfície de folhas, ramos e frutos, dos quais absorve nutrientes através de haustórios que são emitidos para o interior das células epidemias. Segundo BALMER; GALLI (1978), isto implica no fato de que a população do fungo pode estar constantemente se "adaptando" a alterações, resultantes de mutações na população do hospedeiro. Na cultura de trigo, por exemplo, esta doença pode reduzir a área verde capaz de sintetizar carboidratos e desempenhar suas funções. Como uma das consequências, pode provocar redução no número de afilhos, quedas de inflorescência, redução na quantidade e na qualidade de grãos, diminuindo a produtividade (OsórIo,1992). Todavia, ainda não há estudos preci- sos que quantifiquem os prejuízos decorrentes de oídio, para a maioria das culturas de importância econômica no Brasil, incluindo a cultura da soja. Segundo James; Teng (1979), as plantas de soja infectadas com o oídio podem ter a fotossíntese e a transpiração afetadas, e que nos Estados Unidos registrou-se perdas de $10 \%$ a $25 \%$, em campos severamente atacados. Métodos exatos e precisos de avaliação de doenças são fundamentais na quantificação confiável de danos e representa o primeiro passo da pesquisa dentro dessa especialidade.

Com isso o objetivo do trabalho foi quantificar os danos causados por infecção de oídio (M. diffusa) em soja, iniciada nos diferentes estádios fenológicos, em condições de ambiente protegido para auxiliar na definição do melhor momento para estabelecer medidas de controle químico.

\section{MATERIALEMÉTODOS}

Oexperimento foi desenvolvido em condições de telado na Universidade Estadual de Londrina, PR. O delineamento experimental adotado foi o inteiramente casualizado com seis tratamentos e quatro repetições, sendo cada parcela constituída por seis linhas de 2,00 m, espaçadas de 0,40 m entre linhas $(2,0 \mathrm{~m} \mathrm{x}$ 2,0 m). Foi utilizada a cultivar Embrapa 48, classificada como ciclo precoce e suscetível a oídio. Antes da implantação do experimento foram semeadas bordaduras da mesma cultivar, inoculadas artificialmente com oídio (Microsphaera diffusa) após a emergência, através da pulverização sobre as plantas de uma suspensão de esporos, a fim de servirem como fonte de inóculo natural para as respectivas parcelas do experimento posteriormente.

Os tratamentos utilizados foram: testemunha sem controle $\left(\mathrm{T}_{1}\right)$; testemunha controlada $\left(\mathrm{T}_{2}\right)$; infecção iniciada no estádio $\mathrm{R}_{1}-\mathrm{R}_{2}\left(\mathrm{~T}_{3}\right)$; infecção iniciada no estádio $\mathrm{R}_{3}-\mathrm{R}_{4}\left(\mathrm{~T}_{4}\right)$; infecção iniciada no estádio $\mathrm{R}_{5.1}-$ $R_{5.2}\left(T_{5}\right)$ e infecção iniciada no estádio $R_{5.3}-R_{5.4}\left(T_{6}\right)$.

Para possibilitar a obtenção de danos causados por oídio, em que a doença tenha ocorrido em diferentes estádios fenológicos da cultura da soja, estabeleceu-se o seguintes critérios: a) para a testemunha totalmente controlada $\left(\mathrm{T}_{2}\right)$ planejou-se cinco aplicações quinzenais de fungicida epoxiconazole + pyraclostrobin $(0,50$ $\mathrm{L}\left(\mathrm{ha}^{-1}\right)$, iniciando a primeira aplicação no estádio vegetativo $\mathrm{V}_{3} ; \mathrm{b}$ ) para os demais tratamentos estabeleceu-seousodeepoxiconazole + pyraclostrobinna dose de 0,25 L ha-1, distribuídos da seguinte forma: para o tratamento $\mathrm{T}_{3}$, uma aplicaçãono estádio V3; tratamento $\mathrm{T} 4$, duas aplicações nos estádios $\mathrm{V}_{\mathrm{n}} \mathrm{e} \mathrm{R}_{1}$; tratamento $T 5$, três aplicações nos $V_{n^{\prime}} R_{1}$ e $R_{3}$ e para o tratamento $\mathrm{T}$, quatro aplicações nos estádios $\mathrm{V}_{\mathrm{n}^{\prime}}, \mathrm{R}_{1^{\prime}}, \mathrm{R}_{3}, \mathrm{R}_{5.1}-\mathrm{R}_{5.2} \mathrm{e}$ $\mathrm{R}_{5.3}-\mathrm{R}_{5.4^{\prime}}$ conforme demonstrado no Tabela 1. 
Tabela 1 - Esquema de aplicação de fungicida (epoxiconazole + pyraclostrobin) utilizado para proporcionar infecção de oídio em diferentes estádios de desenvolvimento da cultura da soja. Londrina, PR.

\begin{tabular}{lccccccc}
\hline Tratamentos & \multicolumn{4}{c}{ Datas de aplicações de fungicida } & Dose \\
\cline { 2 - 6 } & $22 /$ fev & $02 /$ mar & $09 /$ mar & $16 /$ mar & $30 /$ mar & $15 /$ abr & (L.ha-1 $)$ \\
\hline 01. Testemunha sem controle & - & - & - & - & - & - & - \\
02. Testemunha controlada & + & + & + & + & + & + & 0,50 \\
03. Infecção iniciada nos estádios R1 -R2 & - & + & - & - & - & - & 0,25 \\
04. Infecção iniciada nos estádios R3-R4 & - & + & + & - & - & - & 0,25 \\
05. Infecção iniciada nos estádios R5.1-2 & - & + & + & + & - & - & 0,25 \\
06. Infecção iniciada nos estádios R5.3-4 & - & + & + & + & + & - & 0,25 \\
Estádio Fenológico & V3 & Vn & R1 & R3 & R5.1-2 & R5.3-4 & \\
\hline
\end{tabular}

A avaliação de oídio foi feita semanalmente, considerando a porcentagem da área foliar infectada em três pontos da parcela, utilizando escala adotada por MATTIAZZI (2003). Em cada parcela, avaliou-se um total de 30 plantas, previamente demarcadas.

A colheita foi realizada manualmente, totalizando 30 plantas por repetição (em cada parcela marcou-se cinco plantas por linha, as quais foram avaliadas e colhidas). Nessas foram feitas medição da altura, contagem donúmero de vagens por plantas, pesagem de grãos da parcela (convertido para $\mathrm{kg} \mathrm{ha}^{-1}$ ) e peso de 1.000 sementes.

\section{RESULTADOSEDISCUSSÃO}

Foram observados sintomaticamente, durante a experimentação, outras doenças como a ferrugem $(P$. pachyrhizi) eo mildio (P. manshurica), porém de forma incipiente, uma vez que o desenvolvimento do oídio foi predominante sobre a cultura durante a condução doexperimento.

A análise dos resultados de rendimento $(\mathrm{kg} / \mathrm{ha})$, obtidos na testemunha sem controle (T1), comparada com os da testemunha totalmente controlada, indica que, na cultivar susceptível da soja, o oídio (M. diffusa) é uma doença que pode causar danos significativos na cultura, quando não são estabelecidas medidas de controle adequado (Tabelas 2 e 3 ). Os resultados demonstram também que as perdas na produtividade da soja vão reduzindo gradativamente, na medida em que o estabelecimento da doença ocorre nos estádios mais avançados (Tabela 3).

Os tratamentos em que a infecção iniciou logo aos primeiros estádios, comonos tratamentos $\mathrm{T}_{3} \mathrm{eT}_{4}$ (infecção iniciada em $R_{1}-R_{2}$ e $R_{3}-R_{4}$, respectivamente), apresentaram maiores perdas de produtividade, chegando a se igualarem estatisticamente à testemunha sem controle (Tabela 3). Nesses tratamentos, a produ- tividade foi de 1.186,5 e 1.309,5 kg.ha-1 ${ }^{-1}$ com níveis de perdasno rendimento de $49 \%$ e $44 \%$,respectivamente, em relaçãoà testemunha totalmentecontrolada $(2.337,2$ kg.ha-1), conforme demonstrado na Tabela 3.

Os resultados de perdas de rendimento dos tratamentos $\mathrm{T}_{5} \mathrm{eT}_{6^{\prime}}$ onde a infecção se iniciou nos estádios $R_{5.1}-R_{5.2}$ e $R_{5.3}-R_{5.4^{\prime}}$ não diferiram estatisticamente entre si, tanto para produtividade (1574,2 e 1727,8 kg.ha ${ }^{-1}$ ) comona percentagem de danos de rendimento $(33 \%$ e $26 \%$ ), apresentando porém maiores e menores rendimentos, respectivamente, em relação aos tratamentos $\mathrm{T}_{3} \mathrm{eT}_{4}$ e testemunha totalmente controlada, $\left(2.337,2 \mathrm{~kg}^{\circ} \mathrm{ha}^{-1}\right)$.

Quanto ao número de vagens, houve diferença estatística entre testemunha sem controle, testemunha totalmente controlada e demais tratamentos $\left(\mathrm{T}_{3}, \mathrm{~T}_{4}, \mathrm{~T}_{5} \mathrm{e} \mathrm{T}_{6}\right)$, indicando que o oídio pode também interferir na redução no número de vagens por planta.

Quantoà variável peso de 1.000 sementes, esta não apresentou diferença estatística entre os tratamentos, possivelmente em virtude da dificuldade de manuseiono momento dobeneficiamento, ondeocorrerem perdas das sementes miúdas.

Essa queda na produtividade apresentada pelos tratamentos, de acordo com os níveis de infecção inicial de oídio (M. diffusa), ocorrida nos diferentes estádios fenológicos, se deve ao fato dessa doença causar interferência na assimilação fotossintética da planta. O oídio é uma doença que se desenvolve em toda a parte aérea, formando uma fina cobertura esbranquiçada constituída de micélio e esporos pulverulentos, o que acaba dificultando a fotossíntese, provocando secamento e queda prematura das folhas. Além disso, na infecção severa das hastes e pecíolos, em cultivares susceptíveis, a colonização das células da epiderme das hastes impede a expansão do tecido cortical e, simultaneamente, causa engrossamento do lenho, rachadura das hastes e cicatrizes superficiais (EMBRAPA, 2002). 
Tabela 2 - Área foliar infectada (\%) por oídio (Micosphaera diffusa) na cultura da soja, avaliadas em diferentes estádios fenológicos. Londrina, PR.

\begin{tabular}{|c|c|c|c|c|c|c|c|c|c|}
\hline \multirow[t]{4}{*}{ Tratamentos } & \multicolumn{9}{|c|}{ Porcentagem (\%) da área foliar infectada* } \\
\hline & 1 & 2 & 3 & 4 & 5 & 6 & 7 & 8 & Média \\
\hline & $22 / \mathrm{fev}$ & $2 / \mathrm{mar}$ & $9 / \mathrm{mar}$ & $16 /$ mar & $23 / \operatorname{mar}$ & $30 /$ mar & $6 / \mathrm{abr}$ & $20 / a b r$ & \\
\hline & V3 & Vn & R1 & R2-R3 & R4-R5 & R5.1-2 & R5.3-4 & R7.1 & \\
\hline T1 - Testemunha sem controle & $0,500 \mathrm{~A}$ & $4,000 \mathrm{~A}$ & $20,250 \mathrm{~A}$ & $42,500 \mathrm{~A}$ & $73,750 \mathrm{~A}$ & $90,500 \mathrm{~A}$ & $97,250 \mathrm{~A}$ & $98,750 \mathrm{~A}$ & $53,440 \%$ \\
\hline T2 - Testemunha controlada & $0,001 \mathrm{~B}$ & 0,001 C & 0,001 D & $0,001 \mathrm{E}$ & 0,001 E & 0,001 E & 0,001 D & 0,001 C & $0,001 \%$ \\
\hline $\begin{array}{l}\text { T3 - Infecção iniciada em } \\
\text { R1 - R2 }\end{array}$ & $0,010 \mathrm{~B}$ & 0,050 В & $7,000 \mathrm{~B}$ & 21,250 B & $43,750 \mathrm{~B}$ & $67,500 \mathrm{~B}$ & 86,500 В & $98,000 \mathrm{~A}$ & $40,500 \%$ \\
\hline $\begin{array}{l}\text { T4 - Infecção iniciada em } \\
\text { R3 - R4 }\end{array}$ & 0,010 B & $0,500 \mathrm{~B}$ & $5,750 \mathrm{~B}$ & $17,000 \mathrm{~B}$ & $36,250 \mathrm{~B}$ & $72,500 \mathrm{~B}$ & 81,250 B & $94,500 \mathrm{~A}$ & $38,470 \%$ \\
\hline $\begin{array}{l}\text { T5 - Infecção iniciada em } \\
\text { R5.1 - } 2\end{array}$ & $0,000 \mathrm{~B}$ & 0,080 B & $2,780 \mathrm{C}$ & $11,250 \mathrm{C}$ & $24,500 \mathrm{C}$ & $56,250 \mathrm{C}$ & 80,000 B & $97,000 \mathrm{~A}$ & $33,980 \%$ \\
\hline $\begin{array}{l}\text { T6 - Infecção iniciada em } \\
\text { R5.3 - } 4\end{array}$ & 0,000 B & $0,030 \mathrm{~B}$ & $0,180 \mathrm{D}$ & $3,780 \mathrm{D}$ & $13,750 \mathrm{E}$ & $32,500 \mathrm{D}$ & $58,750 \mathrm{C}$ & 85,000 B & $24,250 \%$ \\
\hline $\mathrm{CV}$ & $39,50 \%$ & $43,15 \%$ & $25,72 \%$ & $17,14 \%$ & $10,10 \%$ & $5,07 \%$ & $5,74 \%$ & $7,52 \%$ & \\
\hline
\end{tabular}

*Média de 30 plantas avaliadas por parcela. Médias seguidas de mesma letra nas colunas não diferem entre si pelo teste Scott Knott a $5 \%$ de probabilidade. Dados transformados em "arcsen $\left((x / 100)^{\wedge} 1 / 2\right)^{\prime}$.

Tabela 3 - Produtividade $\left(\mathrm{kg} \cdot \mathrm{ha}^{-1}\right)$, peso de 1.000 sementes $(\mathrm{g})$, número de vagens por plantas e altura da planta $(\mathrm{cm})$ obtido no experimento. Londrina, PR.

\begin{tabular}{|c|c|c|c|c|c|c|}
\hline \multirow[t]{3}{*}{ Tratamentos } & \multicolumn{6}{|c|}{ Média das avaliações* } \\
\hline & \multirow{2}{*}{$\begin{array}{l}\text { Altura } \\
(\mathrm{cm})^{* *}\end{array}$} & \multirow{2}{*}{$\begin{array}{l}\mathrm{N}^{\circ} \text { total de vagens } \\
\text { por planta** }\end{array}$} & \multirow{2}{*}{$\begin{array}{c}\text { Peso de } \\
1.000 \\
\text { sementes }\end{array}$} & \multicolumn{3}{|c|}{ Produtividade } \\
\hline & & & & $\left(\mathrm{kg} \cdot \mathrm{ha}^{-1}\right)^{* * *}$ & Sc/ha & $\begin{array}{c}\text { Perdas } \\
(\%)\end{array}$ \\
\hline T1 - Testemunha sem controle & $43,54 \mathrm{C}$ & $26,27 \mathrm{C}$ & $110,82 \mathrm{~A}$ & $1167,1 \mathrm{C}$ & 19,5 & 50,1 \\
\hline T2 - Testemunha controlada & $51,22 \mathrm{~A}$ & $40,56 \mathrm{~A}$ & $122,07 \mathrm{~A}$ & $2337,2 \mathrm{~A}$ & 39,0 & - \\
\hline T3 - Infecção iniciada em R1 - R2 & $48,05 \mathrm{~B}$ & 33,66 B & $102,72 \mathrm{~A}$ & $1186,6 \mathrm{C}$ & 19,8 & 49,2 \\
\hline T4 - Infecção iniciada em R3 - R4 & $48,34 \mathrm{~B}$ & $32,53 \mathrm{~B}$ & 104,46 A & $1309,5 \mathrm{C}$ & 21,8 & 44,1 \\
\hline T5 - Infecção iniciada em R5.1 - 2 & 46,83 B & $31,00 \mathrm{~B}$ & $92,33 \mathrm{~A}$ & 1574,2 B & 26,2 & 32,6 \\
\hline T6 - Infecção iniciada em R5.3 - 4 & 47,94 B & $32,07 \mathrm{~B}$ & $97,66 \mathrm{~A}$ & 1727,8 B & 28,8 & 26,1 \\
\hline $\mathrm{CV}$ & $7,31 \%$ & $21,72 \%$ & $15,70 \%$ & & $4,22 \%$ & \\
\hline
\end{tabular}

*Média de 30 plantas avaliadas por parcela. Médias seguidas de mesma letra não diferem entre si pelo teste Scott Knott à $5 \%$ de probabilidade.

**Dados transformados em " $(\mathrm{x}+\mathrm{k})^{\wedge} 1 / 2^{\prime \prime}$ com $\mathrm{k}=1$.

****Dados transformados em " $1 /\left(\mathrm{x}^{\wedge} 1 / 2\right)^{\prime}$.

Nos tratamentos $\mathrm{T}_{3}$ e $\mathrm{T}_{4}$, nos quais a infecção se iniciou nos estádios $R_{1}-R_{2}$ e em $R_{3}-R_{4}$, respectivamente, as plantas estavam no início do florescimento e formação das vagens, estádio este que a infecção diminuiu a área foliar verde provocando redução na quantidade e na qualidade de grãos e, consequentemente, na produtividade. Omesmo ocorreu para os tratamentos $\mathrm{T}_{5}$ e $\mathrm{T}_{6}$, início da infecção nos estádios $R_{5.1}-R_{5.2}$ e $R_{5.3}-R_{5.4^{\prime}}$, respectivamente, influenciando na granação das vagens.
A queda na produção é em razão do estresse causado à planta pelo ataque do fungo, pois folhas infectadas pelo oídio consomem mais do que exportam, tanto em solutos orgânicos como inorgânicos, quando comparadas com folhas sadias. Para compensar a perda de materiais das folhas infectadas, as raízes retiram uma porção maior de fotossintatos doápice da planta. Durante a produção de esporos, um estresse adicional é produzido em toda a planta devido ao grande aumento na perda deágua pelos tecidos ataca- 
dos. Oconjunto dessas alterações pode ter implicações significativas no rendimento ena qualidade dos grãos produzidos posteriormente (MARTINELLI, 2001).

A utilização de fungicida (epoxiconazole + pyraclostrobin) na dose de $0,25 \mathrm{~L} \mathrm{ha}^{-1}$, neste trabalho, teveointuitoderetardaroaparecimentoe/ou evolução do oídio nos respectivos estádios fenológicos para caracterização dos tratamentos estudados. Entretanto, é sabido que as estrobirulinas, neste caso o pyraclostrobin, têm efeitos fisiológicos no tocante ao rendimento das plantas, tais como: aumento na absorção denitrato, inibição da síntese de etileno, ABAe AIA (KoEHLE et al., 2003), o que influenciaria nos níveis de rendimento obtidos. Porém, a evolução acentuada da doença durante a condução do experimento nos respectivos tratamentos denota que o efeito fungistático dofungicida nocontrole dooídio tevepapel preponderante, ao retardar a evolução gerando, consequentemente, redução nas perdas de rendimento.

Sendo assim, verificou-se que, em cultivares suscetíveis como a Embrapa 48, a maior perda na produtividade ocorreu nos tratamentos $\mathrm{T}_{3}$ e $\mathrm{T}_{4^{\prime}}$ quando a planta estava no início do florescimento e na formação das vagens, confirmando a tese de que quanto mais cedo iniciar a infecção, maior será o efeito da doença sobre orendimento (EMBRAPA SOJA, 2002). Fato este devido a área foliar verde ter sido reduzida desde o início do desenvolvimento da cultura, pela infecção do oídioe, assim, as plantas apresentaram reduçãona síntese de carboidratos, ocasionando queda na qualidade e na quantidade de grãos.

Para o tratamento T6, onde a infecção ocorreu no estágio R5.3 (maioria das vagens entre $25 \%$ e $50 \%$ de granação), observou-se a menor média de área foliar infectada pelooídio(24\%), provocando, contudo, queda de $26 \%$ na produtividade(Tabelas $2 \mathrm{e} 3$ ). Tais resultados confrontam com recomendações que vêm sendo feitas para o controle de oídio na cultura da soja, quando recomendam a aplicação de fungicidas à observação de $40 \%$ a $50 \%$ da área foliar atacada pelo oídio (EMBRAPA Soja, 2006). De acordo com os dados obtidos neste estudo, o início do controle químico por meio de fungicidas, quandoosníveisseencontraremaoredorde $40 \%$ a $50 \%$ de área foliar afetada, ocasiona perda de rendimentos com valores próximos a $50 \%$, visto que no T3 (infecção iniciada em R1 - R2) a média de área foliar afetada foide $41 \%$ eaperda foide $49 \%$ na produtividade (Tabelas2e3), oquerepresenta uma perdasignificativa.

Estudos que relacionem as perdas ocasionadas por este patógeno na cultura da soja, correlacionando a infecção em diferentes estádios e sua produção final, não foram encontrados na literatura. Essas informações são úteis para auxiliar na recomendação para o controle deste patógeno, evitando assim a aplicação tardia de fungicidase, consequentemente, redução da produção. Estimativas confiáveis de danos causados pelos patógenos são pré-requisitos para o desenvolvimento de qualquer programa bem sucedido de controle de doenças, independente do método a ser utilizado (JAMES; TENG, 1979). A quantificação de danos causados por doenças é, portanto, um ponto chavena identificação de qualquer estratégia de controle.

\section{CONCLUSÃO}

- A cultivar precoce e suscetível, EMBRAPA 48, às perdas de rendimento pelo oídio (M. diffusa) da soja podem variar ao redor de 26 a $50 \%$.

- A recomendação oficial para o início de controle do oidio ( $M$. diffusa) da soja, quando esta se apresentar entre 40 e $50 \%$ de severidade, deve ser questionada e futuros trabalhos nesteâmbito devem ser desenvolvidos para maiores entendimentos sobre as perdas ocasionadas por esta doença na cultura.

\section{REFERÊNCIAS}

BALMER, E.; GALLI, F. Classificação das doenças segundo a interferência em processos fisiológicos da planta. In: GALLI, F. (Eds). Manual de fitopatologia: princípios e conceitos. 2.ed. São Paulo: Agronômica Ceres, 1978. v.1, p.261-288.

\section{COMPANHIA NACIONAL DO ABASTECIMENTO. \\ (Brasil). Acompanhamento da safra brasileira: grãos: décimo segundo levantamento, setembro 2007 - Brasília, DF, 2007. 24p. Disponível em: <http:/ / www.conab.gov.br/conabweb/download/safra/ 12_levantamento \_se t2007.pdf>. Acesso em: 20 out. 2007.}

EMBRAPA SOJA. (Brasil). Tecnologias de produção de soja região central do Brasil 2003. Londrina: EMBRAPA Soja, 2002. 199p.

EMBRAPA SOJA. (Brasil). Tecnologias de produção de soja - região central do Brasil - 2007. - Londrina: EMBRAPA CNPSo/Embrapa Cerrados/Embrapa Agropecuária Oeste/Embrapa Soja, 2006. n.11. p.169-218.

KOEHLE, H.; GROSSMANN, K.; JABS, T.; GERHARD, M.; KAISER, W.; GLAAB, J.; CONRATH, U.; SEEHAUS, K. \& HERMS,S.2003. Physiological effects of the strobilurin fungicide F 500 on plants. In.: LYR, H.; RUSSEL, P.E.; DEHNE, H.W.; SISLER, H.D. (Ed.). Modern fungicides and antifungal compounds III. Andover: Intercept, 2003.

JAMES, W.C.; TENG, P.S. The quantification of production constraints associated with plant diseases. Applied Biology, n. 4, p.201-267, 1979.

LEHMAN, S.G. Powdery mildew of soybean. Phytopathology, n.37, p.434, 1947. 
MATTIAZZI, P. Efeito do oídio (Microsphaera diffusa Cooke \& Peck) na produção e duração da área foliar sadia da soja. 2003. 49f. Dissertação (Mestrado) - Escola Superior de Agricultura "Luiz de Queiroz", Universidade de São Paulo, Piracicaba, 2003.

MARTINELLI, J. A. Oídios de cereais. In. STADNIK, M.J.; RIVERA, M.C. (Ed.). Oídios. Jaguariúna: Embrapa Meio Ambiente, 2001. p.195-216.

OSÓRIO, E. A. A cultura do trigo. São Paulo: Globo Rural, 1992. p.218. (Coleção do Agricultor - Grãos).
SARTORATO, A.; YORIORI, J. T. Oídios de leguminosas: feijoeiro e soja. In. STADINIK, M.J.; RIVERA, M.C. (Ed.). Oídios. Jaguariúna: Embrapa Meio Ambiente, 2001. p.255-284.

SINCLAIR, J. B. Powdery mildew. In: HARTMAN, G. L.; SINCLAIR, J.B.; RUPE, J.C. (Ed.). Compendium of soybean diseases. 4.ed. St. Paul: APS Press, 1999. 100p.

Recebido em 18/3/09

Aceito em 24/5/10 